\title{
On the Existence of the Real Time Evolution in Euclidean Lattice Gauge Theories
}

\author{
Klaus Fredenhagen * \\ II. Institut für Theoretische Physik, Universität Hamburg, D-2000 Hamburg 50, \\ Federal Republic of Germany
}

\begin{abstract}
A simple argument is given which excludes the occurrence of zero eigenvalues of the transfer matrix in euclidean lattice gauge theories.
\end{abstract}

\section{Introduction}

If the transfer matrix $T$ of a classical statistical mechanics is positive and has no zero eigenvalue, one can associate to this theory a quantum theory where the Hamiltonian $H$ is defined by $H=-\ln T$. The positivity of $T$ is implied by the reflection positivity of the statistical mechanics; euclidean lattice gauge theories have this property for the standard actions $[1,2]$. The absence of zero eigenvalues of $T$ in the thermodynamic limit, however, is an apparently hard problem which remained unsolved over several years [3].

In striking contrast to the difficulties which complicate a direct proof, the solution becomes simple if one formulates the problem in terms of the algebra of observables of the quantum theory. This is possible for finite gauge groups and has first been carried out in [4]. The argument follows:

The local transfer matrices $T_{\mathbf{A}}$ implement (non-*-) automorphisms of the algebra of local observables $\mathfrak{A}$,

$$
\alpha_{i}^{\mathbf{\Lambda}}(A)=T_{\mathbf{\Lambda}} A T_{\mathbf{\Lambda}}^{-1} .
$$

In a theory with finite range interactions these automorphisms converge to a (non-*-) automorphism $\alpha_{i}$ of $\mathfrak{U}$, which may be interpreted as time translation by one unit in imaginary direction.

A ground state $\omega_{0}$ is defined to be a state with

$$
\begin{gathered}
\omega_{0} \alpha_{i}=\omega_{0}, \\
0 \leqq \omega_{0}\left(A^{*} \alpha_{i}(A)\right) \leqq \omega_{0}\left(A^{*} A\right), \quad A \in \mathfrak{A},
\end{gathered}
$$

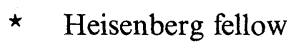


Local ground states (i.e. ground states with respect to $\alpha_{i}^{\mathbf{\Lambda}}$ ) always exist. They are ground states in the usual sense, corresponding to the highest eigenvalue of the local transfer matrix. Their weak limit points for $\Lambda{ }^{\top} \mathbb{Z}^{d}$ fulfill the conditions (1.2), thus also global ground states always exist.

Let $\omega_{0}$ be a ground state in the sense of (1.2). In the GNS-representation $\pi$ of $\mathscr{A}$ with cyclic vector $\Omega$ inducing $\omega_{0}$, one can define the transfer matrix $T$ by

$$
T \pi(A) \Omega=\pi \alpha_{i}(A) \Omega, \quad A \in \mathfrak{A} .
$$

From (1.2), $T$ is a positive contraction with $T \Omega=\Omega$. Since $\alpha_{i}$ is an automorphism of $\mathfrak{U}$ and $\Omega$ is cyclic for $\mathfrak{A}$, the range of $T$ is dense in the representation space $\mathscr{H}_{\pi}$, hence $T$ cannot have a zero eigenvalue. The inverse of $T$ is densely defined and is given by the formula

$$
T^{-1} \pi(A) \Omega=\pi \alpha_{i}^{-1}(A) \Omega, \quad A \in \mathfrak{A} .
$$

The inverse of $\alpha_{i}$. i.e. the translation in negative euclidean time, has no simple counterpart in the statistical mechanics. This might explain why this argument has been overlooked before (as far as I know).

In the case of a continuous gauge group essentially the same argument applies, but the unboundedness of the inverses of the local transfer matrices causes some problems. I treat this case in Sect. 2. In Sect. 3 the equivalence of the algebraic approach with the statistical mechanical approach is shown. In particular it is proven that as a consequence of the DLR equations for the Gibbs state of the statistical mechanics the associated state of the quantum system is locally normal and is a ground state in the sense of Definition (2.17) below.

\section{The Inverse of the Transfer Matrix}

Consider the hypercubic lattice $\mathbb{Z}^{d}, d \geqq 2$ and a compact group $G$, the gauge group. For each box $\boldsymbol{\Lambda}$ in $\mathbb{Z}^{d}, B(\boldsymbol{\Lambda})$ denotes the set of positively oriented bonds and $P(\boldsymbol{\Lambda})$ the set of positively oriented plaquettes in $\Lambda$. The set of local configurations is

$$
G_{\boldsymbol{\Lambda}}=\prod_{b \in B(\boldsymbol{\Lambda})} G_{b},
$$

where for each $b \in B(\boldsymbol{\Lambda}) G_{b}$ is a copy of $G$, and

$$
\mathscr{H}_{\mathbf{\Lambda}}=\mathscr{L}^{2}\left(G_{\boldsymbol{\Lambda}}\right),
$$

the space of square integrable functions on $G_{\boldsymbol{\Lambda}}$ with respect to normalized Haar measure, is the Hilbert space of local state vectors. The algebra of local observables in $\boldsymbol{\Lambda}$ is defined to be the algebra of all bounded operators in $\mathscr{H}_{\mathbf{\Lambda}}$ :

$$
\mathfrak{H}(\mathbf{\Lambda})=\mathscr{B}\left(\mathscr{H}_{\mathbf{\Lambda}}\right) .
$$

If $\boldsymbol{\Lambda}_{1} \subset \boldsymbol{\Lambda}_{2}, \mathfrak{U}\left(\boldsymbol{\Lambda}_{1}\right)$ can be identified with a subalgebra of $\mathfrak{Q}\left(\boldsymbol{\Lambda}_{2}\right)$. Using these identifications one can introduce the algebra of all local observables by

$$
\mathfrak{U}=\bigcup_{\boldsymbol{\Lambda}} \mathfrak{A}(\boldsymbol{\Lambda}) .
$$


The dynamics of the system is characterized by local transfer matrices $T_{\mathbf{\Lambda}} \in \mathfrak{A}(\boldsymbol{\Lambda})$ which stem from, an euclidean action. As operator on $\mathscr{L}^{2}\left(G_{\boldsymbol{\Lambda}}\right), T_{\mathbf{\Lambda}}$ has the following integral kernel

$$
T_{\Lambda}\left(g, g^{\prime}\right)=\exp \left\{\frac{1}{2} \sum_{p \in P(\mathbf{S})}\left[\chi(\partial g(p))+\chi\left(\partial g^{\prime}(p)\right)\right]+\sum_{b \in B(\Lambda)} \chi\left(g(b) g^{\prime}(b)^{-1}\right)\right\},
$$

where $\partial g(p)$ denotes an element in the conjugacy class of the product of $g(b)$ for the bonds $b$ in the boundary of $p$ and where $\chi$ is a real continuous invariant function on $G$ of positive type.

$T_{\mathbf{A}}$ is a positive operator of Hilbert-Schmidt class. It has no zero eigenvalue if and only if in the expansion of $e^{x}$ as a linear combination of simple characters each character occurs with a positive weight. This condition is satisfied e.g. if $\chi$ is a positive multiple of the character of a selfconjugate faithful representation of $G[5]$.

We now want to prove that under this condition also the global transfer matrix has no zero eigenvalue. For this purpose we try to define the imaginary time translation $\alpha_{i}$. Since $T_{\mathbf{\Lambda}}^{-1}$ is not bounded, in general we have to find a suitable domain of definition for $\alpha_{i}$.

First we introduce the (non-*-) automorphism of $\mathfrak{A}$ implemented by the multiplication part of $T_{\mathbf{\Lambda}}$ :



Due to the compactness of the gauge group and the continuity of $\chi, \beta(A)$ exists for all $A \in \mathfrak{A}$.

The convolution part of $T_{\mathbf{A}}$ is also invertible, but the inverse is not bounded in general. Let

$$
e^{x}=\sum_{\xi} c_{\xi} \chi_{\xi}
$$

be the expansion of $e^{x}$ as a linear combination of simple characters. Let $P_{\xi}(b)$ denote the operator of convolution by $\chi_{\xi}(g(b))$ on $\mathscr{H}_{\boldsymbol{\Lambda}}$ for $b \in B(\boldsymbol{\Lambda})$. Then the convolution part $C_{\mathrm{A}}$ of $T_{\mathrm{A}}$ is

$$
C_{\boldsymbol{\Lambda}}=\prod_{b \in \boldsymbol{B}(\boldsymbol{\Lambda})}\left(\sum_{\xi} c_{\xi} P_{\xi}(b)\right),
$$

with the (in general) unbounded inverse

$$
C_{\Lambda}^{-1}=\prod_{b \in B(\Lambda)}\left(\sum_{\xi} c_{\xi}^{-1} P_{\xi}(b)\right) .
$$

Let

$$
\mathfrak{A}_{f}(\boldsymbol{\Lambda})=\bigcup_{P} P \mathfrak{U}(\boldsymbol{\Lambda}) P,
$$

where $P$ runs over the set of projections on the finite dimensional subrepresentations of the left regular representation of $G_{\boldsymbol{\Lambda}}$ on $\mathscr{H}_{\boldsymbol{\Lambda}}$, and let

$$
\mathfrak{A}_{f}=\bigcup_{\boldsymbol{\Lambda}} \mathfrak{A}_{f}(\boldsymbol{\Lambda}) .
$$


For each bond $b$ we define an automorphism $\gamma_{b}$ on $\mathfrak{A}_{f}$ by

$$
\gamma_{b}(A)=\sum_{\xi, \xi^{\prime}} c_{\xi^{\prime}} c_{\xi^{\prime}}^{-1} P_{\xi}(b) A P_{\xi^{\prime}}(b)
$$

If $A \in \mathfrak{U}_{f}(\boldsymbol{\Lambda})$ and $b \notin B(\boldsymbol{\Lambda}), \gamma_{b}$ acts trivially on $A$. Therefore the limit

$$
\gamma(A)=\lim _{\mathbf{\Lambda}>\mathbb{Z}^{d}} \prod_{b \in B(\boldsymbol{\Lambda})} \gamma_{b}(A)
$$

exists for all $A \in \mathfrak{U}_{f}$, and $\gamma$ is a (non-*-) automorphism of $\mathfrak{A}_{f}$. by

Now we can define the imaginary time translation $\alpha_{i}$ for all $A \in \mathscr{D}\left(\alpha_{i}\right)=\beta^{-1}\left(\mathfrak{U}_{f}\right)$

$$
\alpha_{i}(A)=\beta \gamma \beta(A)
$$

Then for each $A \in \mathscr{D}\left(\alpha_{i}\right)$ and for $\boldsymbol{\Lambda}$ sufficiently large we have the relation

$$
\alpha_{i}(A) T_{\mathbf{\Lambda}}=T_{\mathbf{\Lambda}} A,
$$

and $\alpha_{i}(A)$ is uniquely fixed by $(2.15)$.

$\mathscr{D}\left(\alpha_{i}\right)$ is a subalgebra of $\mathfrak{A}$ with the following density property: for each $\Lambda$ there exists some $\Lambda_{1} \supset \boldsymbol{\Lambda}$ such that $\mathfrak{U}(\boldsymbol{\Lambda})$ is contained in the $w^{*}$-closure of $\mathscr{D}\left(\alpha_{i}\right) \cap \mathfrak{A}\left(\boldsymbol{\Lambda}_{1}\right)$, considered as a subalgebra of the von Neumann algebra $\mathfrak{U}\left(\Lambda_{1}\right)$. The inverse $\alpha_{-i}$ of $\alpha_{i}$ has domain $\mathscr{D}\left(\alpha_{-i}\right)=\beta\left(\mathfrak{U}_{f}\right)$ and is defined by

$$
\alpha_{-i}(A)=\beta^{-1} \gamma^{-1} \beta^{-1}(A) \text {. }
$$

$\mathscr{D}\left(\alpha_{-i}\right)$ has the same density property as $\mathscr{D}\left(\alpha_{i}\right)$.

A ground state of $\mathfrak{U}$ with respect to $\alpha_{i}$ is defined to be a locally normal ${ }^{1}$ state $\omega_{0}$ on $\mathfrak{A}$ with

$$
\begin{gathered}
\omega_{0} \alpha_{i}(A)=\omega_{0}(A), \\
0 \leqq \omega_{0}\left(A^{*} \alpha_{i}(A)\right) \leqq \omega_{0}\left(A^{*} A\right),
\end{gathered}
$$

for all $A \in \mathscr{D}\left(\alpha_{i}\right)$. Again, as in the case of finite gauge groups, weak limit points of local ground states fulfill the relations in (2.17). However, it is not clear $a$ priori that these limit points are locally normal. Nevertheless, ground states always exist. This will be shown in Sect. 3.

Let $\omega_{0}$ be a ground state and $(\pi, \mathscr{H}, \Omega)$ the corresponding GNS-representation which is characterized by the relation

$$
(\Omega, \pi(A) \Omega)=\omega_{0}(A), \quad A \in \mathfrak{A} .
$$

Then the transfer matrix $T$ is defined as an operator in $\mathscr{H}$ by

$$
T \pi(A) \Omega=\pi \alpha_{i}(A) \Omega, \quad A \in \mathscr{D}\left(\alpha_{i}\right) .
$$

The density property of $\mathscr{D}\left(\alpha_{i}\right)$ together with the local normality of $\omega_{0}$ implies that $\mathscr{D}\left(\alpha_{i}\right) \Omega$ is dense in $\mathscr{H}$, hence $T$ is densely defined. From (2.15) $T$ is a positive contraction.

1 We recall that a state $\omega$ on $\mathfrak{A}$ is locally normal if for every $\boldsymbol{\Lambda}$ there exists some density matrix $\varrho_{\mathbf{\Lambda}}$ in $\mathscr{H}_{\mathbf{\Lambda}}$ with $\operatorname{Tr} \varrho_{\mathbf{\Lambda}} A=\omega(A)$ for all $A \in \mathfrak{Q}(\mathbf{\Lambda})$ 
That $T$ cannot have an eigenvalue zero is now obvious from the fact that its inverse,

$$
T^{-1} \pi(A) \Omega=\pi \alpha_{-i}(A) \Omega, \quad A \in \mathscr{D}\left(\alpha_{-i}\right),
$$

is densely defined. We arrive at the following theorem.

Theorem. Let $\omega_{0}$ be a ground state of $\mathfrak{A}$ in the sense of Definition (2.17). Then the transfer matrix has no zero eigenvalue.

\section{Equivalence with the Statistical Mechanical Approach}

The euclidean lattice gauge theory corresponding to the quantum system which was introduced in Sect. 2 is defined on the hypercubic lattice $\mathbb{Z}^{d+1}$ with action $S=\sum_{p} \chi(\partial g(p))$. Euclidean fields are continuous functions $f$ of finitely many bond variables $g(b)$. Let $\langle\cdot\rangle$ denote a Gibbs state of the system. This means that $\langle\cdot\rangle$ satisfies the DLR equations $[6,7]$

$$
\langle f\rangle=\left\langle m_{L}(f)\right\rangle
$$

for all euclidean fields $f$ and all finite sets of bonds $L$. Here $m_{L}(f)$ denotes the function

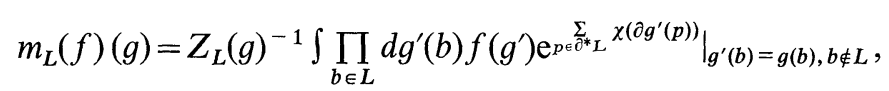

where $\partial^{*} L$ is the set of plaquettes which contain bonds of $L$ in their boundary, and where $Z_{L}(g)$ is fixed by the condition

$$
m_{L}(1)(g)=1 \text {. }
$$

If $f$ depends only on variables $g(b)$ with $b \in L$, then

$$
\sup _{g}\left|m_{L}(f)(g)\right| \leqq \text { const } \int d g|f(g)|,
$$

hence $\langle\cdot\rangle$ can be uniquely extended to $\mathscr{L}^{1}$-functions of finitely many variables. Note that due to $(3.1)\langle\cdot\rangle$ is automatically invariant under gauge transformations [absence of spontaneous breakdown of gauge symmetry (cf. [8])].

A construction of a Hilbert space and a positive transfer matrix is possible if the Gibbs state $\langle\cdot\rangle$ is translation invariant in the 0 -direction and fulfills reflection positivity at the hyperplanes $x^{0}=0$ and $x^{0}=\frac{1}{2}$ in the temporal gauge (see e.g. [9]). This condition can be described in the following way:

Let $\mathscr{F}$ denote the set of euclidean fields which are invariant under gauge transformations at points not contained in the $\left(x^{0}=0\right)$-hyperplane. These functions are fixed by their restrictions to the set $\mathscr{G}_{t}$ of configurations $g$ satisfying the temporal gauge condition $g(b)=1$ for all bonds $b$ pointing in the 0 -direction. In the following we shall identify the functions in $\mathscr{F}$ with their restriction to $\mathscr{G}_{i}$. By $\tau_{n}$ we denote translation in $\mathscr{F}$ by $n$ steps in 0-direction,

$$
\left(\tau_{n} f\right)(g)=f\left(g_{n}\right), \quad g \in \mathscr{G}_{t},
$$


with $g_{n}(b)=g(b+n(1,0))$, and by $\theta$ the operation

$$
(\theta f)(g)=\overline{f\left(g^{g}\right)}, \quad g \in \mathscr{G}_{t},
$$

with $g^{\vartheta}(b)=g(\vartheta(b))$, where $\vartheta$ denotes the reflection through the $\left(x^{0}=0\right)$ hyperplane.

Let $\mathscr{F}_{+} \subset \mathscr{F}$ be the set of fields which depend only on bond variables $g(b)$ with $b$ in the half space $x^{0} \geqq 0$. Then the condition of reflection positivity at hyperplanes $\left(x^{0}=0\right)$ and $\left(x^{0}=\frac{1}{2}\right)$, respectively, reads

$$
\begin{aligned}
\langle(\theta f) f\rangle & \geqq 0, \\
\left\langle(\theta f) \tau_{1} f\right\rangle & \geqq 0,
\end{aligned}
$$

for all $f \in \mathscr{F}_{+}$.

We recall that the physical Hilbert space $\hat{\mathscr{H}}$ is the completion of $\mathscr{F}_{+} / \mathscr{N}$, equipped with the scalar product $(h+\mathscr{N}, f+\mathscr{N})=\langle(\theta h) f\rangle$, where $\mathscr{N}=\left\{f \in \mathscr{F}_{+},\langle(\theta f) f\rangle=0\right\}$, and that the transfer matrix $\hat{T}$ is the positive contraction in $\hat{\mathscr{H}}$ with

$$
\hat{T}(f+\mathcal{N})=\tau_{1} f+\mathscr{N}
$$

(See e.g. [3]).

We now want to show that $\langle\cdot\rangle$ defines a ground state $\omega_{0}$ of the quantum system $\left(\mathfrak{A}, \alpha_{i}\right)$ of Sect. 2 such that there is a unitary operator $U$ from the GNSHilbert space $\mathscr{H}$ onto $\hat{\mathscr{H}}$ such that

$$
\begin{gathered}
U T=\hat{T} U, \\
U \pi(f)=f^{(0)} U,
\end{gathered}
$$

for all multiplication operators $f$, where $f^{(0)}(g)=f\left(g^{(0)}\right), g^{(i)}, i \in \mathbb{Z}$, denotes the restriction of $g$ onto the set of bonds in the $\left(x^{0}=i\right)$-hyperplane, identified with $\mathbb{Z}^{d}$, and $f^{(0)}$ acts by multiplication on $\mathscr{F}_{+} / \mathscr{N}$ (note that $f^{(0)} \mathscr{N} \subset \mathscr{N}$ ).

Let $A \in \mathfrak{U}(\Lambda)$. We associate to $A$ two functions $E_{ \pm}(A) \in \mathscr{F}$ :

$$
\begin{aligned}
& E_{+}(A)(g)=\lim _{\mathbf{\Lambda}_{1} \uparrow \mathbb{Z}^{d}}\left(A T_{\mathbf{\Lambda}_{1}}\right)\left(g^{(0)}, g^{(1)}\right) / T_{\mathbf{\Lambda}_{1}}\left(g^{(0)}, g^{(1)}\right), \\
& E_{-}(A)(g)=\lim _{\boldsymbol{\Lambda}_{1} \ngtr \mathbb{Z}^{d}}\left(T_{\boldsymbol{\Lambda}_{1}} A\right)\left(g^{(-1)}, g^{(0)}\right) / T_{\mathbf{\Lambda}_{1}}\left(g^{(-1)}, g^{(0)}\right) .
\end{aligned}
$$

Due to the finite range of interactions the limits exist and are reached already for a finite $\boldsymbol{\Lambda}_{1}$. Since $T_{\boldsymbol{\Lambda}_{1}}$ is a Hilbert-Schmidt operator in $\mathscr{H}_{\boldsymbol{\Lambda}_{1}}$ with continuous integral kernel, $E_{+}(A)$ and $E_{-}(A)$ are $\mathscr{L}^{2}$-functions. For a multiplication operator $f$, one has the relations

$$
\begin{aligned}
& E_{+}(f A)=f^{(0)} E_{+}(A), \\
& E_{-}(A f)=E_{-}(A) f^{(0)} .
\end{aligned}
$$

We now set

$$
\omega_{0}(A)=\left\langle E_{+}(A)\right\rangle .
$$

$\omega_{0}$ is a locally normal linear functional on $\mathfrak{U}$. For the investigation of its properties we use the following lemma. 
Lemma. Let $A, B \in \mathfrak{U}(\boldsymbol{\Lambda})$, and let $L$ be the set of bonds in $\{0\} \times \Lambda$. Then

$$
\begin{gathered}
\theta E_{+}(A)=E_{-}\left(A^{*}\right), \\
m_{L}\left(E_{+}(A)\right)=m_{L}\left(E_{-}(A)\right), \\
m_{L}\left(E_{+}(A B)\right)=m_{L}\left(E_{-}(A) E_{+}(B)\right), \\
E_{+}\left(\alpha_{i}(A)\right)=\tau_{1} E_{-}(A) .
\end{gathered}
$$

Proof. Let $A\left(g_{0}, g_{0}^{\prime}\right)$ and $B\left(g_{0}, g_{0}^{\prime}\right)$ denote the integral kernels of $A$ and $B$, respectively, considered as operators on $\mathscr{H}_{\mathbf{\Lambda}}$. We use the abbreviations

$$
\begin{gathered}
M\left(g_{0}\right)=\exp \left\{\frac{1}{2} \sum_{p \in \partial^{*} \boldsymbol{B}(\boldsymbol{\Lambda})} \chi\left(\partial g_{0}(p)\right)\right\}, \\
N\left(g_{0}, g_{0}^{\prime}\right)=\exp \left\{\sum_{b \in \boldsymbol{B}(\mathbf{\Lambda})} \chi\left(g_{0}(b) g_{0}^{\prime}(b)^{-1}\right)\right\} .
\end{gathered}
$$

Then for $g \in \mathscr{G}_{t}$,

$$
\begin{aligned}
& E_{+}(A)(g)=\left.\int \prod_{b \in B(\boldsymbol{\Lambda})} d g_{0}(b) A\left(g_{0}, g^{(0)}\right) \cdot \frac{M\left(g_{0}\right)}{M\left(g^{(0)}\right)} \cdot \frac{N\left(g_{0}, g^{(1)}\right)}{N\left(g^{(0)}, g^{(1)}\right)}\right|_{g_{0}(b)=g^{(0)}(b), b \notin B(\mathbf{\Lambda})}, \\
& E_{-}(A)(g)=\left.\int \prod_{b \in B(\mathbf{\Lambda})} d g_{0}(b) A\left(g^{(0)}, g_{0}\right) \cdot \frac{M\left(g_{0}\right)}{M\left(g^{(0)}\right)} \cdot \frac{N\left(g^{(-1)}, g_{0}\right)}{N\left(g^{(-1)}, g_{0}\right)}\right|_{g_{0}(b)=g^{(0)}(b), b \notin B(\boldsymbol{\Lambda})} .
\end{aligned}
$$

Now (i) follows from the fact that the integral kernel of $A^{*}$ is $A^{*}\left(g_{0}, g_{0}^{\prime}\right)=\overline{A\left(g_{0}^{\prime}, g_{0}\right)}$. (ii) is a special case of (iii) $(B=1)$. (iii) The mean $m_{L}$ has the form

$$
m_{L}(f)(g)=\left.\int \prod_{b \in L} d g^{\prime}(b) f\left(g^{\prime}\right) M\left(g^{(0)}\right)^{2} N\left(g^{(-1)}, g^{\prime(0)}\right) N\left(g^{(0)}, g^{(1)}\right)\right|_{g^{\prime}(b)=g(b), b \notin L} .
$$

Inserting for $f E_{+}(A B)$ and $E_{-}(A) E_{+}(B)$, respectively, gives (iii). (iv) follows from (3.10) and (2.15):

$$
\begin{aligned}
E_{+}\left(\alpha_{i}(A)\right)(g) & =\left(\alpha_{i}(A) T_{\mathbf{\Lambda}_{1}}\right)\left(g^{(0)}, g^{(1)}\right) / T_{\mathbf{\Lambda}_{1}}\left(g^{(0)}, g^{(1)}\right) \\
& =\left(T_{\mathbf{\Lambda}_{1}} A\right)\left(g^{(0)}, g^{(1)}\right) / T_{\mathbf{\Lambda}_{1}}\left(g^{(0)}, g^{(1)}\right) \\
& =\tau_{1} E_{-}(A) . \quad \text { q.e.d. }
\end{aligned}
$$

Theorem. $\omega_{0}$ is a ground state of $\mathfrak{U}$ with respect to $\alpha_{i}$.

Proof. Using (3.1), (3.7), (3.12) and the lemma, we find

$$
\omega_{0}\left(A^{*} A\right)=\left\langle E_{+}\left(A^{*} A\right)\right\rangle=\left\langle E_{-}\left(A^{*}\right) E_{+}(A)\right\rangle=\left\langle\left(\theta E_{+}(A)\right) E_{+}(A)\right\rangle \geqq 0,
$$

hence $\omega_{0}$ is a state. For $A \in \mathscr{D}\left(\alpha_{i}\right)$,

$$
\omega_{0} \alpha_{i}(A)=\left\langle E_{+}\left(\alpha_{i}(A)\right)\right\rangle=\left\langle\tau_{1} E_{-}(A)\right\rangle=\left\langle E_{-}(A)\right\rangle=\left\langle E_{+}(A)\right\rangle=\omega_{0}(A),
$$

so $\omega_{0}$ is invariant under $\alpha_{i}$. Furthermore

$$
\begin{aligned}
\omega_{0}\left(A^{*} \alpha_{i}(A)\right) & =\left\langle E_{+}\left(A^{*} \alpha_{i}(A)\right)\right\rangle=\left\langle E_{-}\left(A^{*}\right) E_{+}\left(\alpha_{i}(A)\right)\right\rangle=\left\langle\left(\theta E_{+}(A)\right) \tau_{1} E_{-}(A)\right\rangle \\
& =\left\langle\left(\theta E_{+}(A)\right) \tau_{1} E_{+}(A)\right\rangle=\left(E_{+}(A)+\mathscr{N}, \hat{T}\left(E_{+}(A)+\mathscr{N}\right)\right),
\end{aligned}
$$

where the $4^{\text {th }}$ equality sign follows from the lemma together with (3.1) for the set $L_{1}=\{1\} \times B(\Lambda)$, since $m_{L_{1}}(f h)=f m_{L_{1}}(h)$ if $f$ does not depend on variables $g(b)$ with $b \in L_{1}$. Since $T$ is a positive contraction, $\omega_{0}$ is a ground state. q.e.d. 
We now have the means to prove (3.9). Let

$$
U \pi(A) \Omega=E_{+}(A)+\mathscr{N} .
$$

We have

$$
\begin{aligned}
\|U \pi(A) \Omega\|^{2}=\left\|E_{+}(A)+\mathscr{N}\right\|^{2} & =\left\langle\left(\theta E_{+}(A)\right) E_{+}(A)\right\rangle=\left\langle E_{+}\left(A^{*} A\right)\right\rangle=\omega_{0}\left(A^{*} A\right) \\
& =\|\pi(A) \Omega\|^{2},
\end{aligned}
$$

so $U$ is an isometry. Moreover, for a multiplication operator $f$ from (3.11),

$$
U \pi(f) \pi(A) \Omega=U \pi(f A) \Omega=E_{+}(f A)+\mathscr{N}=f^{(0)} E_{+}(A)+\mathscr{N}=f^{(0)} U \pi(A) \Omega,
$$

hence we have (3.9) (ii). Finally, for $A \in \mathscr{D}\left(\alpha_{i}\right)$,

$$
\begin{aligned}
U T \pi(A) \Omega & =U \pi \alpha_{i}(A) \Omega=E_{+}\left(\alpha_{i}(A)\right)+\mathscr{N}=\tau_{1} E_{-}(A)+\mathscr{N}=\tau_{1} m_{L}\left(E_{-}(A)\right)+\mathscr{N} \\
& =\tau_{1} m_{L}\left(E_{+}(A)\right)+\mathscr{N}=\tau_{1} E_{+}(A)+\mathscr{N} \\
& =\hat{T}\left(E_{+}(A)+\mathscr{N}\right)=\hat{T} U \pi(A) \Omega,
\end{aligned}
$$

thus (3.9) (i) follows from the density of $\pi\left(\mathscr{D}\left(\alpha_{i}\right)\right) \Omega$ in $\mathscr{H}$. It remains to show that $\hat{\mathscr{H}}=U \mathscr{H}$. But $U \mathscr{H}$ is invariant under $\hat{T}$ and under multiplication with time zero fields and contains $(1+\mathscr{N})$, thus $U \mathscr{H}=\hat{\mathscr{H}}$.

\section{Concluding Remarks}

In this paper I treated only the case of a pure gauge theory. It is easy to see that the same methods work in the case where a bounded Higgs field is coupled to the gauge theory. For unbounded Higgs fields and for Fermi fields technical complications may occur which have not been investigated so far.

The absence of zero eigenvalues of the transfer matrix is necessary for the existence of a time evolution of the time zero fields. It does not imply, however, that there is any trace of Einstein causality for this evolution. This is connected with the open question whether the norm closure or at least the weak closure of the quantum algebra of observables is invariant under time evolution. A clarification of these points would be very important for a direct physical interpretation of lattice gauge theories before the continuum limit has been performed.

Acknowledgements. It is a pleasure for me to thank Mihail Marcu, Ricardo Schor and Erhard Seiler for stimulating discussions and Gerhard Mack for critical remarks on the preprint version of this paper.

\section{References}

1. Lüscher, M.: Construction of a selfadjoint, strictly positive transfer matrix for Euclidean lattice gauge theories. Commun. Math. Phys. 54, 283 (1977)

2. Osterwalder, K., Seiler, E.: Gauge field theories on a lattice. Ann. Phys. 110, 440 (1978)

3. Glimm, J., Jaffe, A.: Quantum physics. A functional point of view. New York, Heidelberg, Berlin: Springer 1981

4. Fredenhagen, K., Marcu, M.: Charged states in $\mathbb{Z}_{2}$ gauge theories. Commun. Math. Phys. 92 , 81-119 (1983) 
5. Borgs, C., Seiler, E.: Lattice Yang-Mills theory at nonzero temperature and the confinement problem. Commun. Math. Phys. 91, 329-380 (1983)

6. Dobrushin, R.L.: Description of a random field by means of its conditional probabilities and conditions of its regularity. Theory Probab. Appl. 13, 197-224 (1968)

7. Lanford III, O.E., Ruelle, D.: Observables at infinity and states with short range correlations in statistical mechanics. Commun. Math. Phys. 13, 194-215 (1969)

8. Elitzur, S.: Impossibility of spontaneously breaking local symmetries. Phys. Rev. D 12, 3978-3981 (1975)

9. Seiler, E.: Gauge theories as a problem of constructive quantum field theory and statistical mechanics. Lecture Notes in Physics, Vol. 159. Berlin, Heidelberg, New York: Springer 1982

Communicated by G. Mack

Received April 3, 1985; in revised form May 2, 1985 
